\title{
OPEN Brassica oleracea var. acephala (kale) improvement by biological activity of root endophytic fungi
}

\author{
Jorge Poveda ${ }^{1}$, Iñigo Zabalgogeazcoa ${ }^{2}$, Pilar Soengas ${ }^{1}$, Victor M. Rodríguez ${ }^{1}$, \\ M. Elena Cartea ${ }^{1}$, Rosaura Abilleira ${ }^{1}$ \& Pablo Velasco ${ }^{1}$
}

Brassica oleracea var. acephala (kale) is a cruciferous vegetable widely cultivated for its leaves and flower buds in Atlantic Europe and the Mediterranean area, being a food of great interest as a "superfood" today. Little has been studied about the diversity of endophytic fungi in the Brassica genus, and there are no studies regarding kale. In this study, we made a survey of the diversity of endophytic fungi present in the roots of six different Galician kale local populations. In addition, we investigated whether the presence of endophytes in the roots was beneficial to the plants in terms of growth, cold tolerance, or resistance to bacteria and insects. The fungal isolates obtained belonged to 33 different taxa. Among those, a Fusarium sp. and Pleosporales sp. A between Setophoma and Edenia (called as Setophoma/Edenia) were present in many plants of all five local populations, being possible components of a core kale microbiome. For the first time, several interactions between endophytic fungus and Brassica plants are described and is proved how different interactions are beneficial for the plant. Fusarium sp. and Pleosporales sp. B close to Pyrenophora (called as Pyrenophora) promoted plant growth and increased cold tolerance. On the other hand, isolates of Trichoderma sp., Pleosporales sp. C close to Phialocephala (called as Phialocephala), Fusarium sp., Curvularia sp., Setophoma/Edenia and Acrocalymma sp. were able to activate plant systemic resistance against the bacterial pathogen Xanthomonas campestris. We also observed that Fusarium sp., Curvularia sp. and Setophoma/Edenia confered resistance against Mamestra brassicae larvae.

Brassica crops represent one of the 10 most economically important vegetables in world agricultural and food markets. The most important crop species in this genus are Brassica oleracea (i.e. cauliflower, broccoli, Brussels sprouts, kale, cabbage, ...), Brassica napus (i.e. rapeseed and leaf rape), and Brassica rapa (i.e. turnip, Chinese cabbage, pak choi...), being mainly cultivated in temperate regions of the Northern Hemisphere ${ }^{1}$. Kale, $B$. oleracea var. acephala, is a leafy vegetable which has gained a great popularity as a "superfood" in recent years, due to its anticancerogenic and antioxidant potential associated with the presence of various compounds from the polyphenol, glucosinolate, terpenoid or carotenoid group, and contents of $\mathrm{Ca}$, folate, riboflavin, vitamin $\mathrm{C}$, $\mathrm{K}$ and $\mathrm{A}^{2}$. Furthermore, kale is an important vegetable crop in Iberian Peninsula traditional farming systems, grown for their leaves and flower buds ${ }^{3}$.

The role of microbes in determining the health of soils and plants is increasingly acknowledged. Plants harbour a diversity of microorganisms that may engage in a continuum of interactions ranging from beneficial to adverse interactions. Some of these interactions may be transient and occur during a specific life stage of the plants regardless of its beneficial, detrimental or neutral impact ${ }^{4}$. Endophytes are microbes that can be isolated from asymptomatic plant tissue, including neutral, commensal and/or beneficial microorganisms as well as dormant saprobes and latent pathogens ${ }^{5}$. Some fungal endophytes are well known to contribute to plant fitness, improving the host adaptation to biotic and abiotic stress conditions ${ }^{6}$. For instance, some endophytes are able to reduce the damage of plant pathogens thanks to antagonism via hyperparasitism, competition or antibiosis, or by means of the activation of plant defences ${ }^{7,8}$; others act as entomopathogens (e.g. Beauveria bassiana or Metarhizium anisopliae), protecting host plants against herbivore pests by direct exposure or through the production of insecticidal compounds ${ }^{9}$. Moreover, endophytic fungi are able to increase the plant's tolerance against abiotic stress factors such as drought, salinity or high temperature through the activation of host stress-responses, allowing the plants to avoid or mitigate the impact of the stress ${ }^{10}$. 
As far as the Brassica genus is concerned, there are some studies on the diversity of endophytic fungi and their possible role in improving plant productivity ${ }^{11}$, although none has been done in kale. The inoculation of $B$. napus with Piriformospora indica promoted plant growth, seed yield and quality ${ }^{12}$. Several works focused on the impact of endophytic fungi in the resistance against biotic stresses. Aspergillus flavipes, Chaetomium globosum, Clonostachys rosea and Leptosphaeria biglobosa isolated from B. napus suppressed leaf blight of oilseed rape caused by Sclerotina sclerotiorum ${ }^{13}$. Aspergillus capensis had antifungal activity against the plant pathogenic fungi Botrytis cinerea, Monilinia fructicola, Sclerotinia trifoliorum and S. sclerotiorum ${ }^{14}$. Moreover, the inoculation of B. napus with a Metarhizium anisopliae endophyte provided the plants with greater resistance against Plutella xylostella larvae ${ }^{15}$.

Compared to other plant species, it is considered that the diversity of endophytic fungi present in brassicas is lower than that of other taxonomic groups, probably because of the presence of secondary metabolites derived from tryptophan and other aminoacids, known as glucosinolates ${ }^{16}$. Despite this, the use of endophytic fungi could have an impact on cultivation and the improvement of Brassica-crops within a more environmentally friendly agriculture.

On the other hand, the diversity of endophytic fungi associated with different species within the same genus can be abysmal, and even within the varieties of the same species, as already observed in Uncaria gambier gambir udang and gambir nasi varieties ${ }^{17}$, or in Rosa multiflora and var. carnea ${ }^{18}$.

At present, there are no diversity studies of endophytic fungi present in the roots of B. oleracea and very few in other species of the genus. Therefore, the main objective of this study was to estimate the diversity of root endophytes associated with different accessions of kale. Secondly, we evaluated the biological effect of some dominant endophytes in the promotion of plant growth, tolerance against cold, and resistance against pests and diseases.

\section{Results}

Fungal diversity. From the 900 root-fragments (180 per B. oleracea accession), 376 fungal isolates were obtained, at a rate of 54-98 fungal isolates per accession. Isolates were obtained from $41.77 \%$ of the root fragments plated. All sampled plants harbored fungi in their roots, and on average, 13 isolates were obtained from the roots of each plant.

The isolates were grouped into morphotypes, obtaining 27 morphotypes for MBG-BRS0106, 27 for MBGBRS0292, 45 for MBG-BRS0426, 33 for MBG-BRS0446 and 21 for MBG-BRS0468. After sequencing one or more isolates of each morphotype, 179 ITS1-5.8S-ITS2 nucleotide sequences were obtained. After those sequences were clustered, considering that those being $97 \%$ or more identical belonged to the same taxon, 33 different fungal taxa were identified (Table 1). A phylogenetic tree made with the sequences of these 33 taxa helped to accommodate into taxonomic orders isolates whose sequences were less than $95 \%$ identical to those of a type strain (Supplementary Fig S1).

The most prevalent taxa were a Fusarium sp. found in $63.3 \%$ of the plants collected, and Pleosporales sp. A, found in $56.7 \%$, both were present in all B. oleracea accessions (Table 1). Thanks to the creation of the phylogenetic tree and the comparison of the representative sequence of the proposed taxon Pleosporales sp. A, we have determined how this sequence represents a species not yet described, between the genus Edenia and Setophoma (called from now Setophoma/Edenia). The distribution of the fungal taxa showed few commonalities among the kale accessions (Fig. 1). MBG-BRS0426 and MBG-BRS0468 have 5 fungal taxa in common, 2 of which were not shared with any other accession. Several taxa occurred in only one accession: 10 taxa in MBG-BRS0426, 5 taxa in MBG-BRS0106, 4 taxa in MBG-BRS0292, 4 taxa in MBG-BRS0446, and 3 taxa in MBG-BRS0446. The similarity analysis based on the Jaccard index (JI) (Table 2), shows that only the accessions MBG-BRS0292 and MBG-BRS0446 present a similarity between their fungal taxa greater than 0.3 , while above 0.25 we find MBGBRS0292 and MBG-BRS0468, MBG-BRS0426 and MBG-BRS0468, and MBG-BRS0446 and MBG-BRS0106.

Pleosporales is the most representative order in terms of the number of taxa (32.3\%) (Table 1). Most taxa are present only in one of the accessions (58.8\%), with only $5.9 \%$ of the taxa present in all (Table 1 ). Taking into account the incidence of each order in each variety (Fig. 2), it can be seen that the most abundant order is Pleosporales (27-68\%), followed by Hypocreales (7-34\%), both present in all varieties. Helotiales is present in all accessions, except in MBG-BRS0106, where we find Diaporthales as an specific order. On the other hand, the distribution of taxa according to their incidence can be visualized in the rank-abundance curve shown in Fig. 3. Three taxa were present in more than $45 \%$ of the plants (Fusarium sp. [63.3\%], Setophoma/Edenia [56.7\%] and Acrocalymma sp.[46.7\%]) while the remaining taxa were present in less than $15 \%$ of the plants (Table 1 ).

Increased plant growth, tolerance to cold and resistance to biotic stresses. Nine fungal isolates were used to study beneficial effects on kale. Significant differences in the growth of the aerial part of kale were observed with the application of Fusarium sp. $(5.66 \mathrm{~g})$ and Acrocalymma sp. (4.83 g), being their weight almost double that observed for the control plants without inoculation $(2.92 \mathrm{~g})$. Growth of plants inoculated with Pleosporales sp. B next to Pyrenophora (called from now Pyrenophora) and Curvularia sp. was also significantly higher than that of the control plants. Likewise, although there was no significant difference respect to the control, we observed a reduction in the weight of the plants inoculated with Chaetomium sp. (1.71 g) (Fig. 4a); as well as dry weight (Fig. 4b).

Under cold conditions (constant $12{ }^{\circ} \mathrm{C}$ ) is observed how inoculation with Pyrenophora, Chaetomium sp., Helotiales sp.next to Phialocephala (called from now Phialocephala), Diaporthe sp., Fusarium sp. and Acrocalymma sp. caused a significant increase in growth, and therefore, of tolerance to abiotic stress. Inoculation with Phialocephala, Fusarium sp. and Acrocalymma sp. increased the plant weight almost twice $(2.72 \mathrm{~g}, 2.82 \mathrm{~g}$, and 2.78, respectively) respect to uninoculated control plants (1.47 g) (Fig. 4c); as in dry weight (Fig. 4d). 


\begin{tabular}{|c|c|c|c|c|c|c|}
\hline $\begin{array}{l}\text { Type strain with closest } \\
\text { sequence identity }\end{array}$ & $\begin{array}{l}\text { Identity to closet match } \\
(\%)\end{array}$ & Proposed taxon & $\begin{array}{l}\text { ITS sequence accession } \\
\text { number }\end{array}$ & Order & Incidence in plants (\%) & $\begin{array}{l}\text { Number of } B \text {. oleracea } \\
\text { accesions }\end{array}$ \\
\hline Fusarium foetens & 99.3 & Fusarium sp. & MT628384 & Hypocreales & 63.3 & 5 \\
\hline Edenia gomezpompae & 92.5 & Pleosporales sp. A & MT628351 & Pleosporales & 56.7 & 5 \\
\hline Acrocalymma fici & 95.9 & Acrocalymma sp. & MT626728 & Pleosporales & 46.7 & 3 \\
\hline Alternaria destruens & 100 & Alternaria sp. A & MT628452 & Pleosporales & 20.0 & 3 \\
\hline Pyrenophora nisikadoi & 94.7 & Pleosporales sp. B & MT628399 & Pleosporales & 13.3 & 3 \\
\hline Alternaria mimicula & 100 & Alternaria sp. B & MT628543 & Pleosporales & 13.3 & 3 \\
\hline Barrenia panicia & 98.5 & Barrenia sp. & MT636549 & Helotiales & 13.3 & 3 \\
\hline Ceratobasidium ramicola & 93.1 & Basidiomycota A & MT629733 & unknown & 10.0 & 1 \\
\hline Phialocephala hiberna & 94 & Helotiales sp. & MT628664 & Helotiales & 6.7 & 2 \\
\hline Polyschema sclerotigenum & 98.4 & Polyschema sp. & MT628702 & Pleosporales & 6.7 & 2 \\
\hline Mucor moelleri & 99.1 & Mucor sp. A & MT639934 & Mucorales & 6.7 & 1 \\
\hline Curvularia coatesiae & 99.6 & Curvularia sp. & MT640053 & Pleosporales & 6.7 & 1 \\
\hline Cladosporium spp. & 99.8 & Cladosporium sp. & MT641243 & Capnodiales & 6.7 & 2 \\
\hline Phomopsis tuberivora & 99.4 & Diaporthe sp & MT636064 & Diaporthales & 6.7 & 1 \\
\hline Phoma schachtii & 99.2 & Phoma sp. & MT628903 & Pleosporales & 3.3 & 1 \\
\hline Rhizopus oryzae & 99.8 & Rhizopus sp. & MT635401 & Mucorales & 3.3 & 1 \\
\hline Mucor hiemalis & 96.2 & Mucor sp. B & MT636070 & Mucorales & 3.3 & 1 \\
\hline Penicillium cremeogriseum & 99.8 & Penicillium sp. & MT636161 & Eurotiales & 3.3 & 1 \\
\hline $\begin{array}{l}\text { Chaetomium novozelan- } \\
\text { dicum }\end{array}$ & 99.8 & Chaetomium sp. & MT641231 & Sordariales & 3.3 & 1 \\
\hline Trichoderma hamatum & 99.8 & Trichoderma sp. & MT641233 & Hypocreales & 3.3 & 1 \\
\hline Codinaea acaciae & 98.1 & Codinaea sp. & MT640043 & Chaetosphaeriales & 3.3 & 1 \\
\hline Dendryphion europaeum & 96 & Dendryphion sp. & MT641239 & Pleosporales & 3.3 & 1 \\
\hline Minutisphaera aspera & 99.4 & Minutisphaera sp & MT636088 & Minutisphaerales & 3.3 & 1 \\
\hline $\begin{array}{l}\text { Ceratobasidium papil- } \\
\text { latum }\end{array}$ & 90.7 & Basidiomycota B & MT640104 & unknown & 3.3 & 1 \\
\hline Ceratobasidium ramicola & 91.1 & Basidiomycota C & MT636101 & unknown & 3.3 & 1 \\
\hline $\begin{array}{l}\text { Phragmocephala gareth- } \\
\text { jonesii }\end{array}$ & 92.8 & Pleosporales sp. C & MT641235 & Pleosporales & 3.3 & 1 \\
\hline Trametes versicolor & 99.4 & Trametes sp. & MT635595 & Polyporales & 3.3 & 1 \\
\hline Aaosphaeria arxii & 99 & Aaosphaeria sp. & MT645080 & Pleosporales & 3.3 & 1 \\
\hline Mycofalcella calcarata & 96.9 & Mycofalcella sp. & MT636550 & Helotiales & 3.3 & 1 \\
\hline Aspergillus aureolus & 99.6 & Aspergillus sp. B & MT639933 & Eurotiales & 3.3 & 1 \\
\hline Aspergillus spp. & 98.4 & Aspergillus sp. A & MT641240 & Eurotiales & 3.3 & 1 \\
\hline Tetraploa sasicola & 95.6 & Tetraploa sp. & MT641232 & Pleosporales & 3.3 & 1 \\
\hline $\begin{array}{l}\text { Plectosphaerella niemei- } \\
\text { jerarum }\end{array}$ & 99.4 & Plectosphaerella sp. & MT641266 & Glomerellales & 3.3 & 1 \\
\hline
\end{tabular}

Table 1. Core and abundant fungal species isolated from surface sterilized roots of B. oleracea (kale) from 5 different accessions. A core microbiome is defined as the group of microbes commonly found within a host's microbiome, using persistence of the association as the criterion to select microbes potentially providing critical function within the habitat in which they are found ${ }^{67}$.

Regarding the activation of systemic resistance against biotic stressors, leaves of $B$. oleracea were inoculated with the pathogenic Xcc bacteria (Fig. 5). We observed a significant decrease in the damage caused by the bacteria at 8 d.p.i (Fig. 5a) in the plants inoculated with Trichoderma sp., Phialocephala, Fusarium sp., Curvularia sp., Setophoma/Edenia and Acrocalymma sp., being highly significant $(\mathrm{P}<0.01)$ with the inoculation of the first four fungi indicated. On the other hand, at 15 d.p.i. (Fig. 5b) we only obtained a significant reduction in the incidence of the disease in the plants with the inoculation with the Setophoma/Edenia and Acrocalymma sp. strains.

Regarding the possible activation of systemic resistance in the inoculated plants against the insect pest $M b$, the inoculation of kale with the fungi Setophoma/Edenia (DI 2.3) and Fusarium sp. (DI 2.2) strains supposed a significant decrease in the damage index, compared to the control plants without inoculation (DI 3.1). Furthermore, although no significant differences were observed with respect to the leaf area consumed by the larvae, both Setophoma/Edenia and Fusarium sp. had the lowest average leaf area consumed (Fig. 6).

\section{Discussion}

Few studies have investigated fungal diversity in varieties of the same plant species within a study site. In other words, whether genotypic variation affects the structure of the root microbiome. Regarding the B. oleracea accessions of our study, we have reported a possible specificity of accession. Most of the determined fungal taxa are only present in one accession and the similarity indices give great differences despite the fact that all the plants 


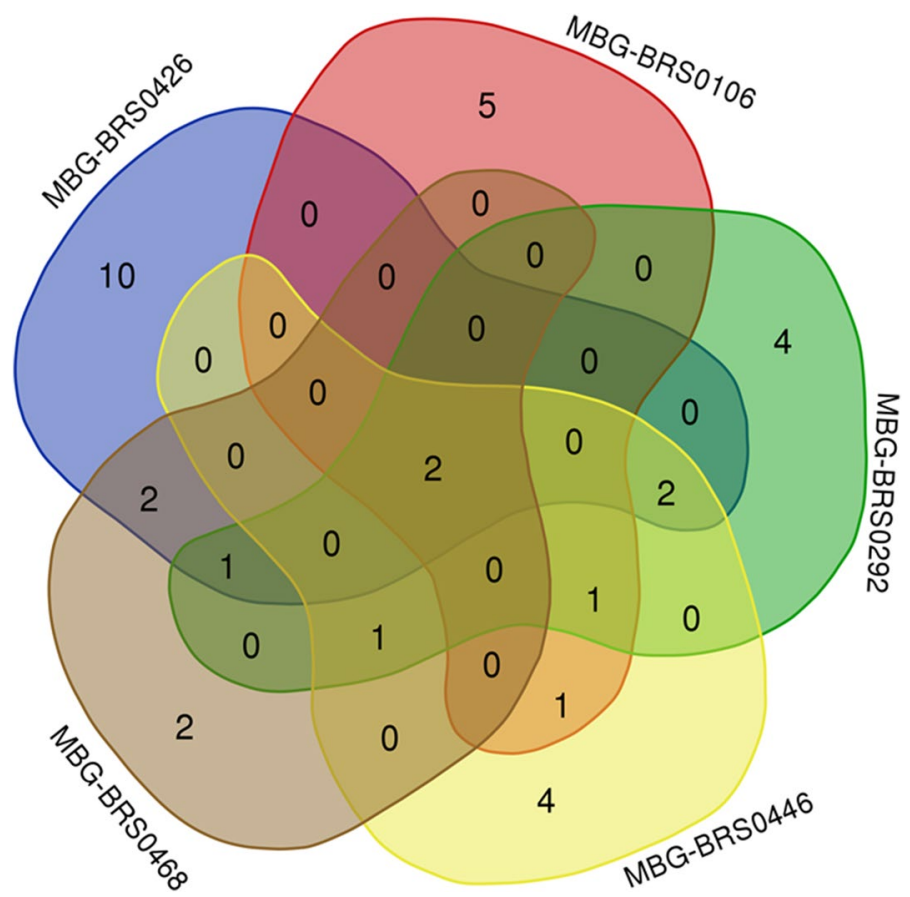

Figure 1. Venn diagram showing the distribution of fungal taxa isolated from the different accessions of $B$. oleracea (kale).

\begin{tabular}{|l|l|l|l|l|l|}
\hline B. oleracea accession & MBG-BRS0426 & MBG-BRS0106 & MBG-BRS0292 & MBG-BRS0446 & MBG-BRS0468 \\
\hline MBG-BRS0426 & $\mathbf{1 . 0 0 0}$ & $\mathbf{0 . 0 8 3}$ & $\mathbf{0 . 2 0 0}$ & $\mathbf{0 . 0 8 3}$ & $\mathbf{0 . 2 5 0}$ \\
\hline MBG-BRS0106 & 24 & $\mathbf{1 . 0 0 0}$ & $\mathbf{0 . 1 7 6}$ & $\mathbf{0 . 2 5 0}$ & $\mathbf{0 . 1 3 3}$ \\
\hline MBG-BRS0292 & 25 & 17 & $\mathbf{1 . 0 0 0}$ & $\mathbf{0 . 3 7 5}$ & $\mathbf{0 . 2 6 7}$ \\
\hline MBG-BRS0446 & 24 & 16 & 16 & $\mathbf{1 . 0 0 0}$ & $\mathbf{0 . 1 8 8}$ \\
\hline MBG-BRS0468 & 20 & 15 & 15 & 16 & $\mathbf{1 . 0 0 0}$ \\
\hline
\end{tabular}

Table 2. Jaccard index of similarity (bold) and total number of fungal taxons identified in roots of each pair of B. oleracea (kale) accessions.

are in the same geographical plot. These differences could be due to differences in the root exudates produced by each kale accession, which could affect the ability of endophytic fungi to colonize the roots, just as it has been observed in different tomato genotypes and their rhizospheric microbiota ${ }^{19}$. Despite this, it is very difficult to consider the existence of such specificity in a study like this, since the possibility of detecting those fungi with low prevalence is more difficult than those that are present to a greater extent, also the great differences existing at the level of alpha (Table 1, Fig. 2) and beta (Table 2) microbial diversity. On the other hand, the presence in all accessions of Fusarium sp. and Setophoma/Edenia, suggests that those two taxa could be components of a core microbiome of kale. This could be confirmed by surveying kale root endophytes at different locations.

The diversity of endophytic fungi present in the roots of plants of the Brassica genus has been scarcely studied so far, as has the diversity of fungi between different varieties of the same species. In our study, most isolated fungi belonged to the Pleosporales order. To our knowledge, only one species of the Pleosporales order, Leptosphaeria biglobosa, has been found in B. napus ${ }^{13}$. Besides, order Hypocreales was found in all accessions. This order is more common in Brassicaceae, and taxa such as Fusarium sp. ${ }^{20}$, C. rosea ${ }^{13}$ and M. anisopliae ${ }^{15}$ have been reported in B. napus. Mucorales order was found in 3 of the 5 accessions, having previously being reported Mucor sp. in $B$. campestris $^{21}$ and B. chinensis ${ }^{22}$. Sordariales order was only found in MBG-BRS0292 and MBG-BRS0468 accessions, having previously being described the fungus Chaetomium globosum in B. napus roots ${ }^{13}$. And Eurotiales order was only found in the MBG-BRS0446 accession, having previously reported the fungus Penicillium sp. $.^{20}, A$. flavipes $^{13}$ and A. capensis ${ }^{14}$ in B. napus roots. Therefore, our study reports the first description of the Helotiales, Capnodiales, Diaporthales, Chaetosphaeriales and Minutisphaerales orders in roots of a plant of the Brassica genus; Heliotales order was widely present in 4 of the 5 accessions.

In our study carried out with different accessions of B. oleracea (kale), we have been able to determine the presence of two dominant taxa in all the accessions examined (Fusarium sp. and Setophoma/Edenia). Species of the genus Fusarium, such as F. oxysporum can be neutral, beneficial, or detrimental for host plants. Fusarium wilt is one of the most devastating diseases in agriculture, since some strains can also cause vascular wilts resulting 
MBG-BRS0426

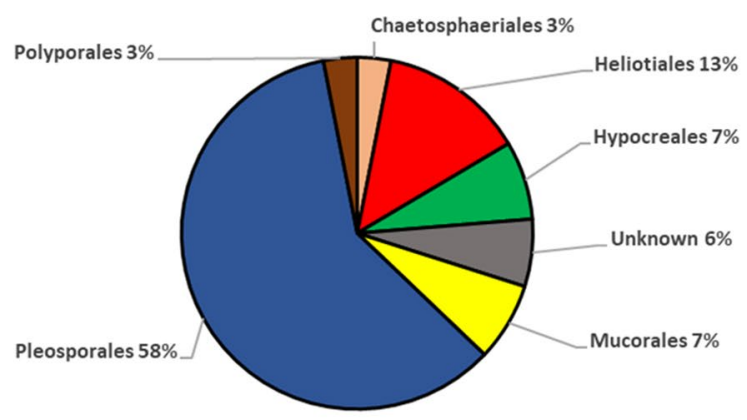

MBG-BRS0106

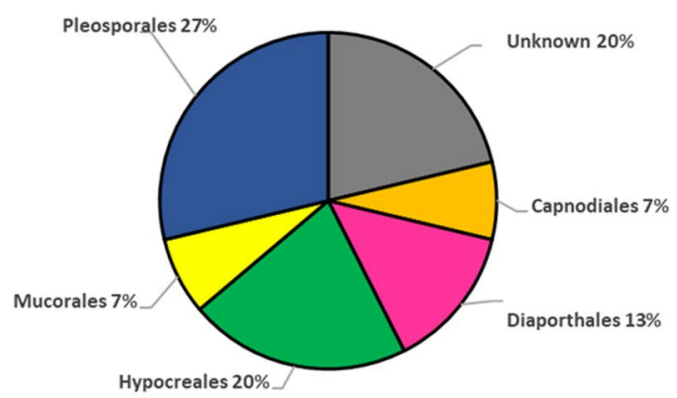

MBG-BRS0292

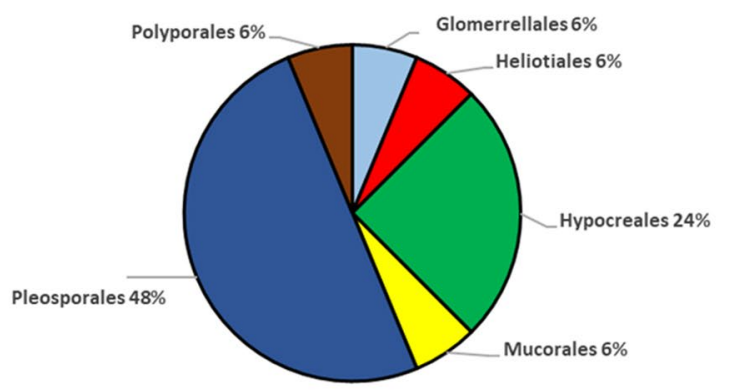

MBG-BRS0446

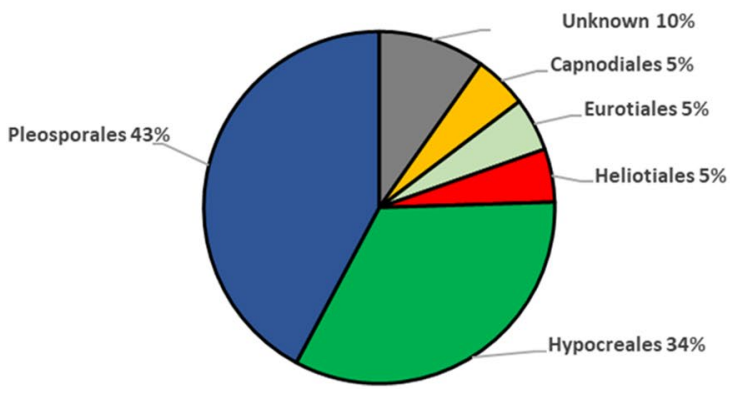

MBG-BRS0468

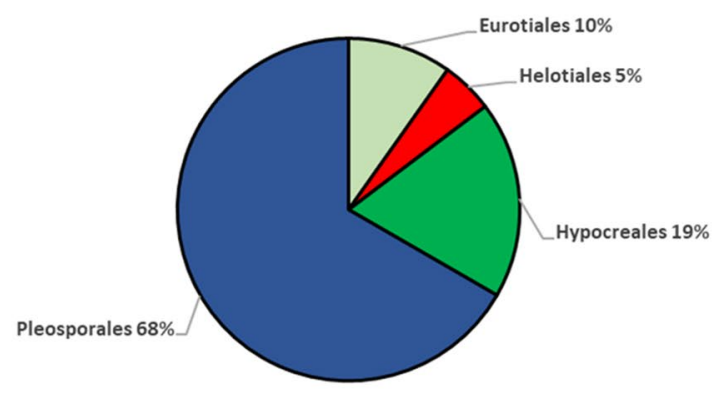

Figure 2. Distribution of fungal taxa from roots of different accessions of $B$. oleracea (kale) according to orders.

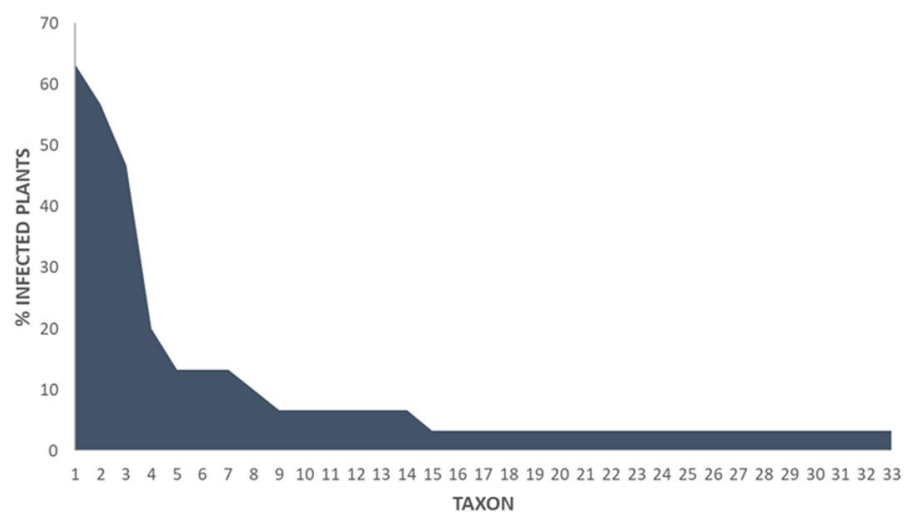

Figure 3. Rank-abundance plot showing the incidence in plants of each taxon identified in roots of B. oleracea (kale).

in serious yield losses in affected crops. Despite this, numerous strains of F. oxysporum behave as endophytes capable of activating plant systemic resistance against the pathogenic strains of the fungus ${ }^{23}$, or produce protective secondary metabolites ${ }^{24}$, like other species such as F. tricinctum ${ }^{25}$. Nevertheless, all the existing studies with F. oxysporum f. sp. conglutinans describe it as a pathogen of the Brassica genus, for example, studying the transcriptome profiles in different lines of $B$. rapa after its inoculation ${ }^{26}$, or transcriptomic and proteomic studies 
(a)

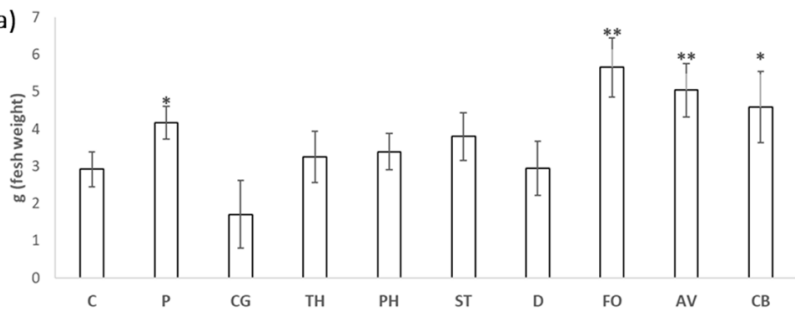

(c) 4

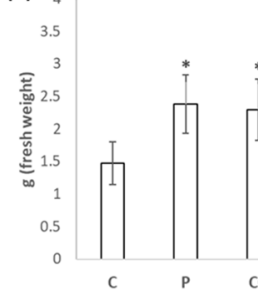

(b)

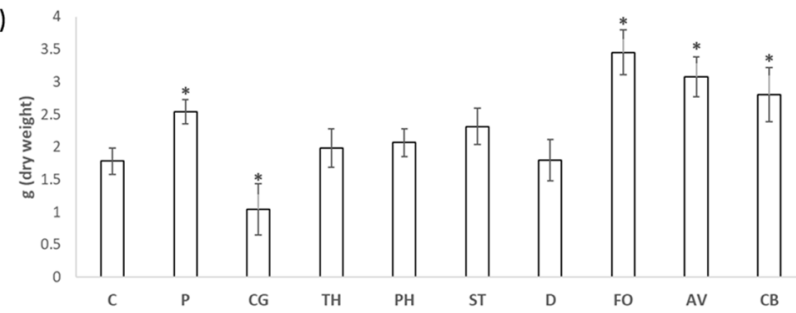

(d)

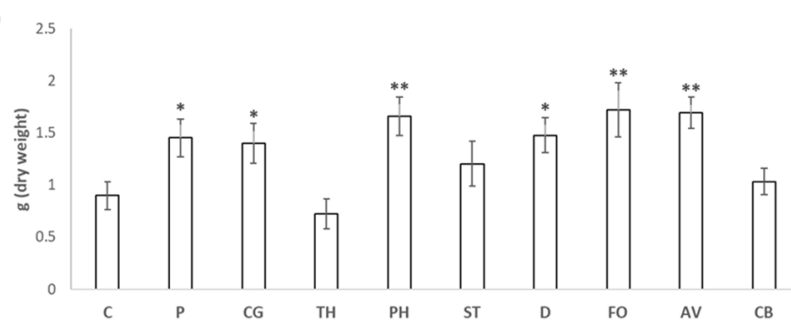

Figure 4. Mean of fresh $(\mathbf{a}, \mathbf{c})$ and dry weight $(\mathbf{b}, \mathbf{d})$ of kale plants grown in greenhouse $(\mathbf{a}, \mathbf{b})$ and cold $(\mathbf{c}, \mathbf{d})$ conditions. Plants without inoculation (C) and inoculated with unknown Pyrenophora (P), Chaetomium sp. (CG), Trichoderma sp. (TH), Phialocephala (PH), Setophoma/Edenia (ST), Diaporthe sp. (D), Fusarium sp. (FO), Acrocalymma sp. (AV) and Curvularia sp. (CB) were collected at 12-week-old and measured their fresh weight of the aerial part. Data are the mean of 25 plants for each inoculation with the corresponding standard deviation. Student's t-test was performed. Asterisks denote significant differences at $P \leq 0.05\left(^{\star}\right)$ and $P \leq 0.01$ ${ }^{* *}$.
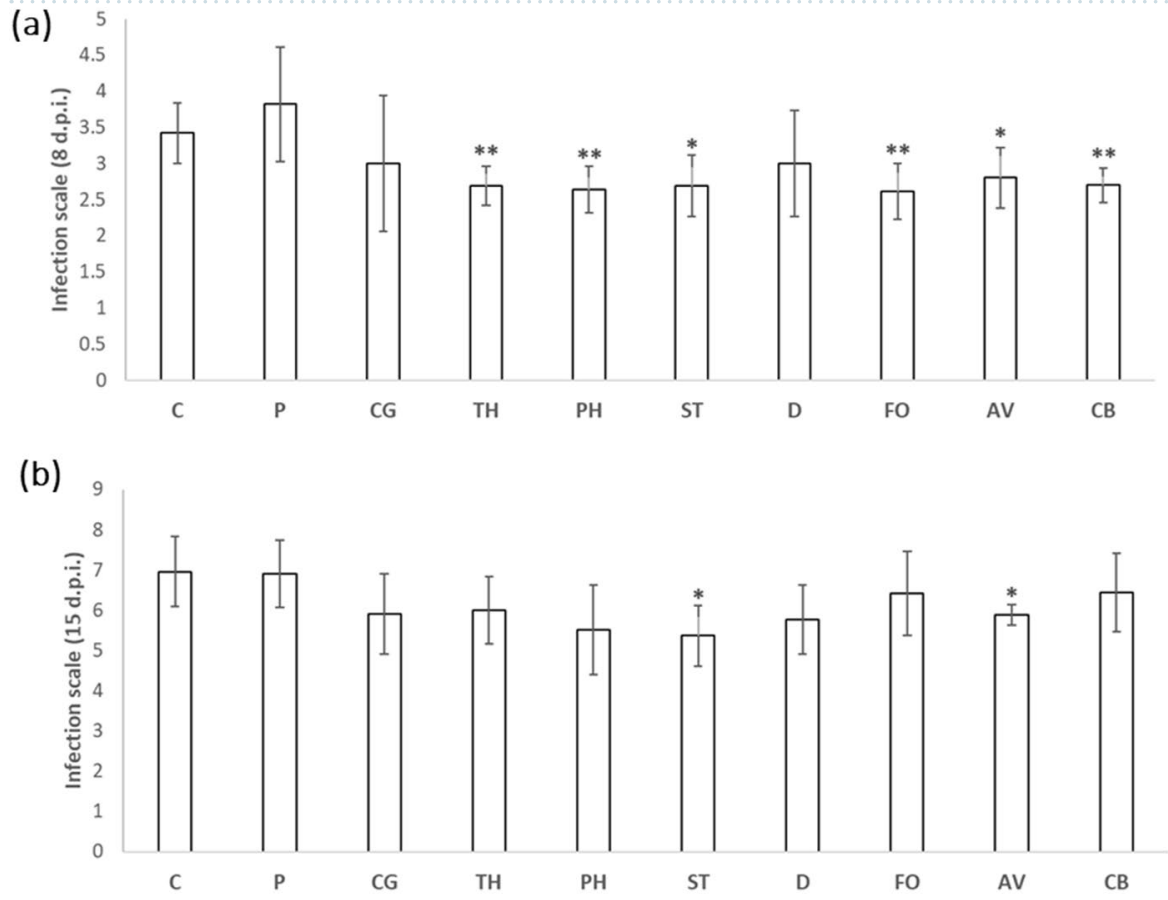

Figure 5. Effect of $X c c$ infection on kale plants inoculated with endophytic fungi. Plants without inoculation (C) and inoculated with unknown Pyrenophora (P), Chaetomium sp. (CG), Trichoderma sp. (TH), Phialocephala (PH), Setophoma/Edenia (ST), Diaporthe sp. (D), Fusarium sp. (FO), Acrocalymma sp. (AV) and Curvularia sp. (CB) were infected at 5-6 leaves stage and the leaves with lesions are classified according to an infection range from 1 to 5 . Data are the mean 10 plants for each inoculation with the corresponding standard deviation. Student's t-test was performed. Asterisks denote significant differences at $\left.P \leq 0.05{ }^{(}\right)$and $P \leq 0.01\left(^{(*}\right)$.

in B. oleracea ${ }^{27,28}$. So, this is the first study that describes Fusarium as an endophyte of brassica roots, although Fusarium oxysporum and other species of the genus have been described as dominant components of the microbiome of several plant species ${ }^{29}$.

As regards the taxa Setophoma/Edenia, there is information about pathogenic and endophytic species in both genus. Setophoma includes disease-causing pathogenic fungal species such as leaf spot in Camellia sinensis 
(a)

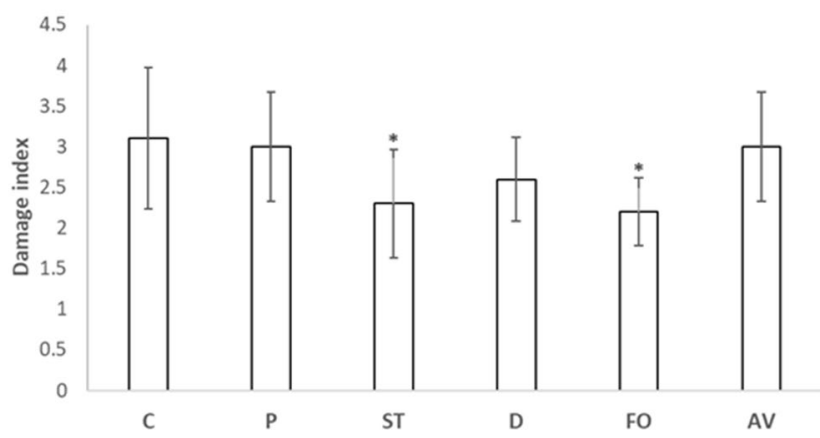

(b)

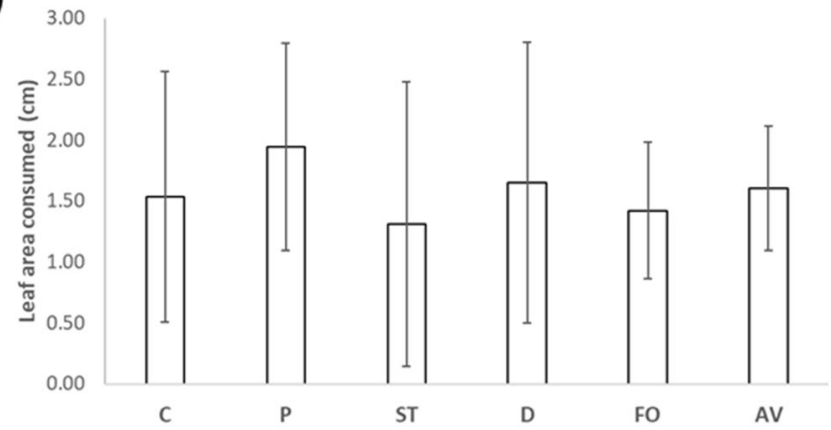

Figure 6. Effect of infestation with M. brassicae on kale plants inoculated with endophytic fungi. Plants without inoculation (C) and inoculated with Pyrenophora (P), Setophoma/Edenia (ST), Diaporthe sp. (D), Fusarium sp. (FO) and Acrocalymma sp. (AV) were attacked at 12-week-old, obtaining 5 d.p.a. the damage index in a range from 1 to 5 and the leaf area consumed by the larvae. Data are the mean of 10 plants for each inoculation with the corresponding standard deviation, and for each biological replicate and condition, three leaves/plants were used. Student’s t-test was performed. Asterisks denote significant differences at $P \leq 0.05\left(^{\star}\right)$.

(S. antiqua, S. longinqua, S. yingyisheniae and S. yunnanensis) ${ }^{30}$, but mainly to root level. S. terrestris (formerly called Phoma terrestris) causes pink root rot on members of the Allium genus and other crops, such as tomato, eggplant, pepper, maize and carrot. In 2017, the first case of pink root rot in the Brassica genus was described in B. napus ${ }^{31}$, being the only report of $S$. terrestris-Brassica interaction described so far. On the other hand, $S$. terrestris has been described as an endophyte of other plant species such as Gloriosa superba ${ }^{32}$ and horseradish (Armoracia rusticana), where is able to decompose various glucosinolates ${ }^{33}$. In turn, Edenia is a well-known genus of endophytic fungi that produce compounds of high medical interest such as anti-inflammatories ${ }^{34}$ or antileshmanials $^{35}$. Furthermore, the E. gomezpompae species is capable of producing highly toxic compounds for plants, which are used as natural herbicides ${ }^{36}$. Both within the Setophoma genus and the Edenia genus, our isolate represents the first endophyte identified in roots of Brassica plants.

An Acrocalymma sp. was present in 3 of the 5 accessions studied. A. medicaginis has been described as a causal agent of crown and root rot in Medicago plants ${ }^{37}$. However, A. vagum is a dark septate endophyte able to decrease heavy metal content in tobacco ${ }^{38}$ and to promote plant growth in Glycyrrhiza uralensis ${ }^{39}$, but never before reported in Brassica plants.

Therefore, in the present study we have been able to identify several taxa of endophytic fungi in kale roots, which had not been described so far as endophytic fungi (although in some cases as pathogens) within the Brassica genus.

Once the different endophytic fungi in the kale roots were identified, we made a selection of them to determine their possible biological role in the plant. To do this, we inoculated kale plants and tried to determine if the endophytes were capable of promoting plant growth, increasing cold tolerance, or inducing systemic plant resistance against pathogens and/or pests.

The ability of different species of endophytes to promote plant growth as well as tolerance to abiotic stresses such as cold, or resistance to pathogens has been described ${ }^{40}$. In our study, we have observed that the Pyrenophora strain was capable of promoting the growth of the plant and its cold tolerance. This would be the first time that a benefit of the Pyrenophora-plant interaction has been described, this genus is best known for pathogens, such as Pyrenophora teres, causal agent of net blotch of barley ${ }^{41}$. The beneficial role observed in B. oleracea by Pyrenophora could be due to the defensive capacity of cruciferous plants through secondary metabolites such as glucosinolates, not present in other plant groups, which is why a possible pathogen in a species may be a harmless symbiont in Brassica. Moreover, inoculation with Fusarium sp. in B. oleracea also increased its growth and cold tolerance, more significantly than with Pyrenophora. Although there are numerous studies that demonstrate 
the ability of non-pathogenic strains of Fusarium to increase the resistance of plants against various pathogens, such as F. oxysporum ${ }^{23}$, their role as a possible promoter of plant growth or cold tolerance has not been studied.

The interaction B. oleracea-Acrocalymma sp. resulted in a significant increase in plant growth, something previously observed in other plants such as Glycyrrhiza uralensis ${ }^{39}$, Medicago sativa and Ammopiptanthus mongolicus $^{42}$ in interaction with A. vagum. Similar results have been reported in B. oleracea-Curvularia sp. interaction, fungal genus that, although it includes important plant pathogens ${ }^{43}$, also has species classified as endophytes that promote plant growth, such as C. geniculata in Parthenium hysterophorus through phosphate solubilization and phytohormone production ${ }^{44}$.

Regarding the increase in cold tolerance, we have observed that the B. oleracea-Chaetomium sp. interaction results in a significant benefit for the plant. In this sense, several authors have described different mechanisms such as heat shock proteins or antioxidant compounds that, for example, the endophyte C. globosum has to tolerate cold, some of which may also be beneficial for the plant thanks to the interaction ${ }^{45-47}$. The interaction of $B$. oleracea with Phialocephala also produced an increase in plant tolerance to cold, as has been verified in different subarctic herbaceous plants by Phialocephala fortinii ${ }^{48}$. Also, we have observed the capacity of Diaporthe sp. and Acrocalymma sp. to increase the tolerance of $B$. oleracea to cold, being the first time, even within their fungal genera, that these beneficial capacities for plants have been described.

After infection with $X c c$, we quantified a decrease in the harmful effect of the bacteria on B. oleracea plants preinoculated with Trichoderma sp. The ability of different Trichoderma species to activate plant systemic resistance against different pathogens has been widely proven in various plant species ${ }^{49}$, including Brassica plants as B. napus with T. harzianum ${ }^{50,51}$ or T. viride ${ }^{52}$, B. rapa with T. pseudokoningii ${ }^{53}$, and even B. oleracea var. capitate with T. harzianum against Rhizoctonia solani ${ }^{54}$.

As far as the Fusarium sp. strain is concerned, the ability of F. oxysporum endophytic strains to induce systemic plant resistance has been described in zucchini plants (Cucurbita pepo) against the insect Aphis gossypii ${ }^{55}$, in banana plants (Musa spp.) against the nematode Radopholus similis ${ }^{56}$, in Asparagus officinalis against the pathogen F. oxysporum f. sp. asparagi $i^{57}$, and in tomato plants against F. oxysporum f. sp. lycopersici ${ }^{58}$. While in the case of inoculation with Phialocephala, Curvularia sp., Setophoma/Edenia and Acrocalymma sp., the data obtained represents the first report of activation of plant systemic resistance by these fungi as root endophytes.

As conclusions, the diversity of endophytic fungi found in the kale roots of various accessions is lesser compared to non-brassicaceae species, where up to 49 fungal taxa can be found per plant ${ }^{59}$. In addition, several of the isolated fungal taxa have been widely described as pathogens of different crops, but never as endophytes, as species of the genus Pyrenophora, an aspect that occurs in kale probably due to their powerful defensive capacity through the use of glucosinolates against fungi ${ }^{60}$. On the other hand, we find taxa present in several of the accessions and even in all of them, as is the case of Fusarium sp. and Setophoma/Edenia, representing a possible core microbiome for kale. It is precisely these taxa that have shown in our preliminary studies a greater capacity to promote the productivity of kale, by promoting its growth, increasing its tolerance to cold and increasing its defensive capacity against pathogens and pests.

\section{Materials and methods}

Plant material, crop and sampling. Five kale accessions were used in this study. These are local populations from Galicia (Northwestern Spain), kept in the Brassica Germplasm Collection of the Misión Biológica de Galicia (MBG-CSIC). The codes for these accessions are MBG-BRS0106, MBG-BRS0292, MBG-BRS0426, MBG-BRS0446 and MBG-BRS0468.

The study was conducted in 2016 at MBG-CSIC (Galicia, NW Spain). Two hundred seeds of each accession were sown in trays in May 2016, and kept in a greenhouse under environmental conditions. After 50 days, when most seedlings were at the 4-5 leaf stage, one hundred and fifty plants of each accession were transplanted into an experimental plot randomly distributed. The distance was $0.8 \mathrm{~m}$ between rows and $0.6 \mathrm{~m}$ between plants. The experimental plot was established in a slightly acidic soil (pH close to 5.5), organic matter content of 6.3\%, $100 \mathrm{ppm}$ available $\mathrm{P}, 335 \mathrm{ppm}$ assimilable $\mathrm{K}, 133 \mathrm{ppm}$ changeable $\mathrm{Mg}$, and $7.83 \mathrm{cmol} / \mathrm{kg} \mathrm{Ca}{ }^{2+}$. As management practices, no fungicide was applied, or any other pesticide, or any form of fertilization. To prevent the development of weeds, the soil was covered with a mesh that prevents their presence in the plot.

Sampling was carried out in 30-week-old plants, randomly choosing 6 plants per accession. After digging out the entire root system, samples of the roots were collected, and stored in cold for a few hours until processing in the laboratory.

Isolation of fungi. A subsample of 30 root fragments of $4-5 \mathrm{~cm}$ per plant was collected. Disinfection of roots and fungal isolation was done following the procedure described by Ref. ${ }^{29}$. Each root sample was washed with tap water and then surface-disinfected with a solution of $20 \%$ commercial bleach ( $1 \%$ active chlorine) containing $0.02 \%$ Tween 80 (v:v) for $6 \mathrm{~min}$, followed by treatment with an aqueous solution of $70 \%$ ethanol for $30 \mathrm{~s}$. Finally, the roots were rinsed with sterile water and cut into pieces about $5 \mathrm{~cm}$ long. Thirty root pieces of each sample were plated in three Petri plates (10 pieces/plate) with potato dextrose agar (PDA) (Sigma-Aldrich, St. Louis, USA) containing $200 \mathrm{mg} / \mathrm{L}$ of chloramphenicol (the antibiotic was used to avoid the isolation of endophytic bacteria), and kept in the dark at room temperature. As mycelium emerged from a root fragment into the agar, a small piece of the mycelium from the leading edge of the colony was transferred to a new PDA plate and maintained at room temperature. The root fragment and remaining mycelium were taken out of the original plate to avoid overgrowth. The plates with root samples were checked daily for the presence of fungi for about 4 weeks. 


\begin{tabular}{|l|l|l|l|}
\hline Proposed taxon & Name in the text & Abbreviation & Presence in kale accessions \\
\hline Pleosporales sp. B (next to Pyrenophora) & Pyrenophora & P & $\begin{array}{l}\text { MBG-BRS0292 } \\
\text { MBG-BRS0446 } \\
\text { MBG-BRS0468 }\end{array}$ \\
\hline Chaetomium sp. & Chaetomium sp. & CG & MBG-BRS0292 \\
\hline Trichoderma sp. & Trichoderma sp. & TH & MBG-BRS0446 \\
\hline Helotiales sp. (next to Phialocephala) & Phialocephala & PH & $\begin{array}{l}\text { MBG-BRS0426 } \\
\text { MBG-BRS0468 }\end{array}$ \\
\hline Pleosporales sp. A (next to Setophoma and Edenia) & Setophoma/Edenia & ST & All \\
\hline Diaporthe sp. & Diaporthe sp. & D & MBG-BRS0106 \\
\hline Fusarium sp. & Fusarium sp. & FO & All \\
\hline Acrocalymma sp. & Acrocalymma sp. & AV & $\begin{array}{l}\text { MBG-BRS0426 } \\
\text { MBG-BRS0292 } \\
\text { MBG-BRS0468 }\end{array}$ \\
\hline Curvularia sp. & Curvularia sp. & CB & MBG-BRS0426 \\
\hline
\end{tabular}

Table 3. Fungal isolates used in kale inoculation.

Identification of fungi and diversity analysis. The fungal isolates obtained from roots were first grouped into different morphotypes according to morphological characteristics such as colony color, exudate production, mycelium appearance, and growth rate $^{29}$. One or a few isolates of each morphotype were used for further classification and identification based on rDNA nucleotide sequences. Fungal DNA was extracted from a small amount of mycelium scraped from a PDA culture using the Phire Plant Direct PCR Kit (Thermo Fisher Scientific). A ribosomal DNA region including the internal transcribed spacer 1 (ITS1), 5.8S rDNA, and ITS2 was amplified by PCR using primers ITS1 and ITS $4^{61}$. Amplification conditions were: $98{ }^{\circ} \mathrm{C}$ for 5 min, followed by 35 cycles of $98^{\circ} \mathrm{C}$ for $5 \mathrm{~s}, 54^{\circ} \mathrm{C}$ for $5 \mathrm{~s}$, and $72{ }^{\circ} \mathrm{C}$ for $20 \mathrm{~s}$; after that the reaction was kept at $72{ }^{\circ} \mathrm{C}$ for $1 \mathrm{~min}$. PCR amplicons were cleaned (MSB Spin PCRapace, Stratec biomedical, Germany) and sequenced at the Genomics Service in the CACTI, University of Vigo, Spain (https://cactiweb.webs.uvigo.es/).

All the sequences obtained were grouped into operational taxonomic units (OTU), considering that groups of sequences with a similarity greater than $97 \%$ belonged to the same OTU. This clustering operation was done using CD-Hit-Est software ${ }^{62}$. For taxonomic identification, a representative sequence of each OTU was used to search the GenBank nucleotide database using BLAST. This search was limited to sequences belonging to the ITS region from fungi type and reference material. Taxonomic identification was limited to genus rank because often occurs that ITS sequences are not accurate for species rank identification (i.e. Ref. ${ }^{30}$ ). When the identity between our sequences and that of a type strain was lower than $95 \%$, the genus of our sequence was considered to be unknown. Further taxonomic information was obtained by means of a phylogenetic tree made with representative sequences from each taxon. This tree was made with MEGA6 software using the maximum likelihood method with distances calculated according to the Tamura 3 parameter model ${ }^{63}$. Tree branch confidence values were estimated by bootstrapping with 1000 replications.

For each kale accession, the incidence of each fungal taxon in plants was calculated, relating the number of plants in which it was found against the total of plants sampled (30 root fragments per plant, 6 plants per accession, 30 plants in total), as alpha diversity analysis. Additionally, the distribution of the relative abundance of each taxon was observed with a rank-abundance curve. Furthermore, beta diversity was analyzed by comparing between accessions, using the Jaccard index.

In planta assays. Plant-fungus interaction. One of the local varieties previously used in the diversity study (MBG-BRS0106, from here on referred to as kale) was used for the reinoculation tests and the study of the biological activity of the selected fungi.

Different fungal isolates (Table 3) were chosen based on their presence in all accessions, or their proven biological activity in other plant species (bibliographic search). To inoculate plants, one part of beet pulp inoculum was mixed with seven parts (w/w) of a substrate consisting of peat moss (Profi-Substract, Gramoflor, Valencia, Spain) previously treated at $80^{\circ} \mathrm{C}$ for $24 \mathrm{~h}$. The fungal inoculum was a 4 -week-old culture of each fungus grown in sugar beet pulp medium ${ }^{64}$. Uninoculated controls were transplanted to soil mixed with uninoculated beet pulp medium. By qPCR, the ability of fungi to effectively colonize kale roots could be verified (data not shown).

Growth promotion. For the analysis of plant growth promotion kale plants were inoculated in $20 \mathrm{~L}$ pots. The plants were kept under controlled greenhouse conditions until their aerial part was harvested in 8-week-oldplants to record fresh weight. The plants were watered 2-3 times a week, according to the observed needs, always with the same amount of water in all the plants. Exogenous fertilization was not used. Greenhouse conditions were $14 \mathrm{~h}$ photoperiod, environmental temperature $\left(12-30^{\circ} \mathrm{C}\right)$ and relative humidity above $80 \%$. A total of 25 plants were used for each fungal isolate inoculated.

Cold tolerance. For the cold tolerance assay, we stablished the same conditions reported in Ref. ${ }^{65}$. Seeds of kale were planted in $1 \mathrm{~L}$ pots and grown under fluorescent light $\left(228 \mu \mathrm{mol} \mathrm{m}{ }^{-2} \mathrm{~s}^{-1}\right)$ in a $14 \mathrm{~h} \mathrm{light} / 10 \mathrm{~h}$ dark photoperiod regime and watered as needed. The temperature in the cold-exposure treatment was set at $12 \pm 1^{\circ} \mathrm{C}$, since 
lower temperatures reduced seed germination and seedling survival dramatically. A total of 25 plants were used for each inoculation.

Biotic resistance. Inoculation of kale leaves with the bacterial plant pathogen Xanthomonas campestris pv. campestris $(\mathrm{Xcc})$ was made according to Ref. ${ }^{66}$. Bacterial cultures were grown in PDA at $30{ }^{\circ} \mathrm{C}$ for $48 \mathrm{~h}$. A bacterial suspension was diluted with sterile water to reach a turbidity value of 0.5 , which corresponds to a concentration of $5 \times 10^{8} \mathrm{cfu} / \mathrm{mL}$. Turbidity of the suspension was measured with a microplate spectrophotometer (Spectra MR; DynexTechnologies, Chantilly, VA) at a wavelength of $600 \mathrm{~nm}$. The second leaf counting from the apex was inoculated when plants were at a 5-6 leaf stage. Inoculation was made by biting on three veins located on the edge of the leaf with a mouse-tooth forceps wrapped in cotton submerged in the inoculum. Xcc race 1 type strain HRI3811, provided by Joana Vicente (University of Warwick, UK), was used to inoculate the plants. After the inoculation, plants were kept under controlled greenhouse conditions $\left(20^{\circ} \mathrm{C}\right.$ at night and $28^{\circ} \mathrm{C}$ during the day, and relative humidity greater than $80 \%$ ). Damage was measured using a visual damage index (DI) scale ranging from 1 to 9 , where $1=$ no visible symptoms and $9=$ severely diseased with typical $V$-shaped chlorotic leaf edge lesions presenting blackened veins areas. Data were taken 8 and 11 days post infection (d.p.i.). A total of 10 plants were used for each fungal strain.

Infestation with the insect pest Mamestra brassicae $(\mathrm{Mb})$ was carried out under the controlled greenhouse conditions above described using 12-week-old-plants. Eggs were provided by the Laboratory of Entomology, Wageningen University, The Netherlands. Egg hatching larvae were fed with fresh leaves of B. oleracea (MBGBRS0106) ad libitum. $M b$ inoculation was carried out depositing 5 7-10 days old larvae on the 5-6th true leaf of the plant. After 5 days, data on leaf area consumed per plant and the damage index were recorded: $1=$ no damage, $2=1-10 \%$ leaf consumed, $3=11-20 \%$ leaf consumed, $4=21-30 \%$ leaf consumed, $5=$ more than $30 \%$ of the leaf consumed. A total of 10 plants were used for each fungal strain.

Statistical analysis. For each trait (i.e. growth promotion, tolerance to cold, inoculation with $X c c$ and infestation with $\mathrm{Mb}$ ) a Student's t-test was used to compare the means of each fungal inoculation treatment with the control at $\mathrm{P}<0.05$ and $\mathrm{P}<0.01$; significant differences are denoted using asterisks.

Received: 24 August 2020; Accepted: 6 November 2020

Published online: 19 November 2020

\section{References}

1. Francisco, M. et al. Nutritional and phytochemical value of Brassica crops from the agri-food perspective. Ann. Appl. Biol. 170, 273-285 (2017).

2. Šamec, D., Urlić, B. \& Salopek-Sondi, B. Kale (Brassica oleracea var. acephala) as a superfood: Review of the scientific evidence behind the statement. Crit. Rev. Food Sci. Nutr. 59, 2411-2422 (2019).

3. Velasco, P., Cartea, M. E., González, C., Vilar, M. \& Ordás, A. Factors affecting the glucosinolate content of kale (Brassica oleracea acephala group). J. Agric. Food Chem. 55, 955-962 (2017).

4. Schouten, A. Endophytic fungi: Definitions, diversity, distribution and their significance in plant life. In Endophyte Biotechnology: Potential for Agriculture and Pharmacology. 6-31 (CABI, 2019).

5. Compant, S., Saikkonen, K., Mitter, B., Campisano, A. \& Mercado-Blanco, J. Editorial special issue: Soil, plants and endophytes. Plant Soil 405, 1-11 (2016).

6. Kumar, V., Soni, R., Jain, L., Dash, B. \& Goel, R. Endophytic Fungi: Recent Advances in Identification and Explorations. In Advances in Endophytic Fungal Research 267-281 (. Springer, Cham, 2019).

7. Busby, P. E., Ridout, M. \& Newcombe, G. Fungal endophytes: Modifiers of plant disease. Plant Mol. Biol. 90, 645-655 (2016).

8. Poveda, J., Abril-Urias, P. \& Escobar, C. Biological control of plant-parasitic nematodes by filamentous fungi inducers of resistance: Trichoderma, mycorrhizal and endophytic fungi. Front. Microbiol. 11, 992 (2020).

9. Quesada-Moraga, E., Herrero, N., Zabalgogeazcoa, Í. Entomopathogenic and nematophagous fungal endophytes. In Advances in Endophytic Research. 85-99 (Springer, New Delhi, 2014).

10. Lata, R., Chowdhury, S., Gond, S. K. \& White, J. F. Jr. Induction of abiotic stress tolerance in plants by endophytic microbes. Lett. Appl. Microbiol. 66, 268-276 (2018).

11. Card, S. D. et al. Beneficial endophytic microorganisms of Brassica-A review. Biol. Control 90, 102-112 (2015).

12. Su, Z. Z. et al. Piriformospora indica promotes growth, seed yield and quality of Brassica napus L. Microbiol. Res. 199, 29-39 (2017).

13. Zhang, Q. et al. Diversity and biocontrol potential of endophytic fungi in Brassica napus. Biol. Control 72, 98-108 (2014).

14. Qin, J. et al. Strain identification and metabolites isolation of Aspergillus capensis CanS-34A from Brassica napus. Mol. Biol. Rep. 46, 3451-3460 (2019).

15. Batta, Y. A. Efficacy of endophytic and applied Metarhizium anisopliae (Metch.) Sorokin (Ascomycota: Hypocreales) against larvae of Plutella xylostella L. (Yponomeutidae: Lepidoptera) infesting Brassica napus plants. Crop Prot. 44, 128-134 (2013).

16. Hiruma, K., Kobae, Y. \& Toju, H. Beneficial associations between Brassicaceae plants and fungal endophytes under nutrient-limiting conditions: Evolutionary origins and host-symbiont molecular mechanisms. Curr. Opin. Plant Biol. 44, 145-154 (2018).

17. Ilyas, M., Kanti, A., Jamal, Y., Hertina, H. \& Agusta, A. Biodiversity of endophytic fungi associated with Uncaria gambier Roxb. (Rubiaceae) from west Sumatra. Biodiversitas 10, 2009 (2009).

18. Zhao, Y. et al. Fungal endophytic communities of two wild Rosa varieties with different powdery mildew susceptibilities. Front. Microbiol. 9, 2462 (2018).

19. Kovacs, E. D. et al. Rhizosphere microbiota profile changes with different genetic types of tomato species. Agricultura 109, 140-150 (2019).

20. Shi, Y. et al. Effects of Cd-and Pb-resistant endophytic fungi on growth and phytoextraction of Brassica napus in metal-contaminated soils. Environ. Sci. Pollut. Res. 24, 417-426 (2017).

21. Zahoor, M. et al. Alleviation of heavy metal toxicity and phytostimulation of Brassica campestris L. by endophytic Mucor sp. MHR7. Ecotox. Environ. Saf. 142, 139-149 (2017).

22. Deng, Z. et al. Characterization of Cd-and Pb-resistant fungal endophyte Mucor sp. CBRF59 isolated from rapes (Brassica chinensis) in a metal-contaminated soil. J. Hazard. Mater. 185, 717-724 (2011). 
23. de Lamo, F. J. \& Takken, F. L. Biocontrol by Fusarium oxysporum using endophyte-mediated resistance. Front. Plant Sci. 11, 37 (2020).

24. Caicedo, N. H., Davalos, A. F., Puente, P. A., Rodríguez, A. Y. \& Caicedo, P. A. Antioxidant activity of exo-metabolites produced by Fusarium oxysporum: An endophytic fungus isolated from leaves of Otoba gracilipes. Microbiol. Open 8, e903 (2019).

25. Ola, A. R., Thomy, D., Lai, D., Brötz-Oesterhelt, H. \& Proksch, P. Inducing secondary metabolite production by the endophytic fungus Fusarium tricinctum through coculture with Bacillus subtilis. J. Nat. Prod. 76, 2094-2099 (2013).

26. Miyaji, N. et al. Comparison of transcriptome profiles by Fusarium oxysporum inoculation between Fusarium yellows resistant and susceptible lines in Brassica rapa L. Plant Cell. Rep. 36, 1841-1854 (2017).

27. Pu, Z. et al. Changes in the proteome of xylem sap in Brassica oleracea in response to Fusarium oxysporum stress. Front. Plant Sci. 7, 31 (2016).

28. Xing, M. et al. Transcriptome profiling of resistance to Fusarium oxysporum f. sp. conglutinans in cabbage (Brassica oleracea) roots. PLoS ONE 11, e0148048 (2016).

29. Pereira, E., Vázquez de Aldana, B. R., San Emeterio, L. \& Zabalgogeazcoa, I. A survey of culturable fungal endophytes from Festuca rubra subsp. pruinosa, a grass from marine cliffs, reveals a core microbiome. Front. Microbiol. 9, 3321 (2019).

30. Liu, F., Wang, J., Li, H., Wang, W. \& Cai, L. Setophoma spp. on Camellia sinensis. Fungal Syst. Evol. 4, 43-57 (2019).

31. Yang, Y. et al. First report of pink root rot caused by Setophoma (Pyrenochaeta) terrestris on canola. Can. J. Plant. Pathol. 39, $354-360$ (2017).

32. Shobha, M., Kumara, K. S. \& Prakash, H. S. Fungal endophytes associated with Gloriosa superba (L.). Proc. Natl Acad. Sci. India Sect. B Biol. Sci. 89, 1335-1342 (2019).

33. Szücs, Z. et al. Endophytic fungi from the roots of horseradish (Armoracia rusticana) and their interactions with the defensive metabolites of the glucosinolate-myrosinase-isothiocyanate system. BMC Plant Biol. 18, 85 (2018).

34. Tan, Y. et al. Anti-inflammatory spirobisnaphthalene natural products from a plant-derived endophytic fungus Edenia gomezpompae. Chin. Chem. Lett. 31, 1406-1409 (2020).

35. Martínez-Luis, S. et al. Antileishmanial constituents of the Panamanian endophytic fungus Edenia sp. J. Nat. Prod. 71, 2011-2014 (2008).

36. Macias-Rubalcava, M. L., Ruiz-Velasco Sobrino, M. E., Melendez-Gonzalez, C. \& Hernandez-Ortega, S. Naphthoquinone spiroketals and organic extracts from the endophytic fungus Edenia gomezpompae as potential herbicides. J. Agric. Food Chem. 62, 3553-3562 (2014).

37. Irwin, J. A. G., Mackie, J. M., Marney, T. S., Musial, J. M. \& Roberts, S. Incidence of Stagonaspora meliloti and Acracalymma medicaginis in lucerne crowns and roots in eastern Australia, their comparative aggressiveness to lucerne and inheritance of reaction to S. meliloti in lucerne. Aust. Plant Pathol. 33, 61-67 (2004).

38. Jin, H. Q. et al. Effect of the dark septate endophytic fungus Acrocalymma vagum on heavy metal content in tobacco leaves. Symbiosis 74, 89-95 (2018).

39. He, C., Wang, W. \& Hou, J. Characterization of dark septate endophytic fungi and improve the performance of liquorice under organic residue treatment. Front. Microbiol. 10, 1364 (2019).

40. Singh, B. P. Advances in Endophytic Fungal Research (Springer, New York, 2019).

41. Clare, S. J., Wyatt, N. A., Brueggeman, R. S. \& Friesen, T. L. Research advances in the Pyrenophora teres-barley interaction. Mol. Plant. Pathol. 21, 272-288 (2020).

42. Hou, L., Yu, J., Zhao, L. \& He, X. Dark septate endophytes improve the growth and tolerance of Medicago sativa and Ammopiptanthus mongolicus under cadmium stress. Front. Microbiol. 10, 3061 (2019).

43. Bengyella, L. et al. Biotechnological application of endophytic filamentous bipolaris and curvularia: A review on bioeconomy impact. World J. Microbiol. Biotechnol. 35, 69 (2019).

44. Priyadharsini, P. \& Muthukumar, T. The root endophytic fungus Curvularia geniculata from Parthenium hysterophorus roots improves plant growth through phosphate solubilization and phytohormone production. Fungal Ecol. 27, 69-77 (2017).

45. Liu, Z. H., Yang, Q. \& Nie, Y. H. Cloning and expression of Hsp22.4 gene from Chaetomium globosum. J. For. Res. 17, 259-262 (2006).

46. Liu, Z. H., Yang, Q. \& Ma, J. A heat shock protein gene (hsp22.4) from Chaetomium globosum confers heat and $\mathrm{Na}_{2} \mathrm{CO}_{3}$ tolerance to yeast. Appl. Microbiol. Biotechnol. 77, 901-908 (2007).

47. Wang, Z. et al. Antioxidant activity of a polysaccharide produced by Chaetomium globosum CGMCC 6882. Int. J. Biol. Macromol. 141, 955-960 (2019).

48. Acuña-Rodríguez, I. S., Newsham, K. K., Gundel, P. E., Torres-Díaz, C. \& Molina-Montenegro, M. A. Functional roles of microbial symbionts in plant cold tolerance. Ecol. Lett. 23, 1034-1048 (2020).

49. Poveda, J., Eugui, D. \& Abril-Urías, P. Could be Trichoderma a plant pathogen? Succesful of colonization. In: Trichoderma: Host Pathogen Interactions and Applications. 35-39 (Springer, New York, 2020).

50. Alkooranee, J. T. et al. Systemic resistance to powdery mildew in Brassica napus (AACC) and Raphanus alboglabra (RRCC) by Trichoderma harzianum TH12. PLoS ONE 10, e0142177 (2015).

51. Poveda, J., Hermosa, R., Monte, E. \& Nicolás, C. The Trichoderma harzianum Kelch protein ThKEL1 plays a key role in root colonization and the induction of systemic defense in Brassicaceae plants. Front. Plant Sci. 10, 1478 (2019).

52. Alkooranee, J. T., Aledan, T. R., Xiang, J., Lu, G. \& Li, M. Induced systemic resistance in two genotypes of Brassica napus (AACC) and Raphanus oleracea (RRCC) by Trichoderma isolates against Sclerotinia sclerotiorum. Am. J. Plant Sci. 6, 1662 (2015).

53. Li, H. Y. et al. Trichokonins from Trichoderma pseudokoningii SMF2 induce resistance against Gram-negative Pectobacterium carotovorum subsp. carotovorum in Chinese cabbage. FEMS Microbiol. Lett. 354, 75-82 (2014).

54. Shibu, M. A., Lin, H. S., Yang, H. H. \& Peng, K. C. Trichoderma harzianum ETS 323-mediated resistance in Brassica oleracea var capitata to Rhizoctonia solani involves the novel expression of a glutathione S-transferase and a deoxycytidine deaminase. J. Agric. Food Chem. 60, 10723-10732 (2012).

55. Martinuz, A., Schouten, A., Menjivar, R. D. \& Sikora, R. A. Effectiveness of systemic resistance toward Aphis gossypii (Hom., Aphididae) as induced by combined applications of the endophytes Fusarium oxysporum Fo162 and Rhizobium etli G12. Biol. Control 62, 206-212 (2012).

56. Paparu, P., Dubois, T., Coyne, D. \& Viljoen, A. Defense-related gene expression in susceptible and tolerant bananas (Musa spp.) following inoculation with non-pathogenic Fusarium oxysporum endophytes and challenge with Radopholus similis. Physiol. Mol. Plant Pathol. 71, 149-157 (2007).

57. He, C. Y., Hsiang, T. \& Wolyn, D. J. Induction of systemic disease resistance and pathogen defence responses in Asparagus officinalis inoculated with nonpathogenic strains of Fusarium oxysporum. Plant Pathol. 51, 225-230 (2002).

58. Constantin, M. E., de Lamo, F. J., Vlieger, B. V., Rep, M. \& Takken, F. L. Endophyte-mediated resistance in tomato to Fusarium oxysporum is independent of ET, JA, and SA. Front. Plant Sci. 10, 79 (2019).

59. Vandenkoornhuyse, P., Baldauf, S. L., Leyval, C., Straczek, J. \& Young, J. P. W. Extensive fungal diversity in plant roots. Science 295, 2051-2051 (2002).

60. Poveda, J., Eugui, D. \& Velasco, P. Natural control of plant pathogens through glucosinolates: An effective strategy against fungi and oomycetes. Phytochem. Rev. 161, 1-15 (2020).

61. White, T. J., Bruns, T., Lee, S. J. W. T., Taylor, J. Amplification and direct sequencing of fungal ribosomal rna genes for phylogenetics. In PCR Protocols: a Guide to Methods and Applications. 315-322 (Academic Press, Cambridge, 1990). 
62. Li, W. \& Godzik, A. Cd-hit: A fast program for clustering and comparing large sets of protein or nucleotide sequences. Bioinformatics 22, 1658-1659 (2006) https://weizhongli-lab.org/cd-hit/.

63. Tamura, K., Stecher, G., Peterson, D., Filipski, A. \& Kumar, S. MEGA6: Molecular evolutionary genetics analysis version 6.0. Mol. Biol. Evol. 30, 2725-2729 (2013).

64. Vázquez-de-Aldana, B. R., Cuesta, M. J. \& Zabalgogeazcoa, I. Cultivation and growth dynamics of endophytic fungi in a solid culture medium based on sugar beet pulp. J. Sci. Food Agric. 100, 441-446 (2020).

65. Rodríguez, V. M. et al. Effect of temperature stress on the early vegetative development of Brassica oleracea L. BMC Plant. Biol. 15, 145 (2015).

66. Iglesias-Bernabé, L., Madloo, P., Rodríguez, V. M., Francisco, M. \& Soengas, P. Dissecting quantitative resistance to Xanthomonas campestris pv. campestris in leaves of Brassica oleracea by QTL analysis. Sci. Rep. 9, 1-11 (2019).

67. Hernandez-Agreda, A., Gates, R. D. \& Ainsworth, T. D. Defining the core microbiome in corals' microbial soup. Trends Microbiol. 25, 125-140 (2017).

\section{Acknowledgements}

This research was financially supported by project RTI2018-096591-B-I00 34 (MCIU/AEI/FEDER, UE). JP has a contract from the Xunta de Galicia IN607A2016/13. Authors want to thank Dr. Rieta Gols (Laboratory of Entomology, Wageningen University, Wageningen, The Netherlands) for providing us with Mamestra eggs and for the unvaluable inputs in this manuscript.

\section{Author contributions}

P.V. conceived the study. P.V., J.P. and I.Z. discussed the results. P.S. carried out the Xcc experiment. M.E.C. carried out the $M b$ experiment. V.M.R. carried out the cold experiment and the molecular analyses. R.A. maintained the fungal collection. J.P. and P.V. performed statistical analysis of the data. I.Z. identified the fungal sequences. J.P. drafted the initial manuscript. All authors read, edited and approved the final version of the manuscript.

\section{Competing interests}

The authors declare no competing interests.

Additional information

Supplementary information is available for this paper at https://doi.org/10.1038/s41598-020-77215-7.

Correspondence and requests for materials should be addressed to P.V.

Reprints and permissions information is available at www.nature.com/reprints.

Publisher's note Springer Nature remains neutral with regard to jurisdictional claims in published maps and institutional affiliations.

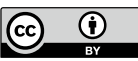

Open Access This article is licensed under a Creative Commons Attribution 4.0 International License, which permits use, sharing, adaptation, distribution and reproduction in any medium or format, as long as you give appropriate credit to the original author(s) and the source, provide a link to the Creative Commons licence, and indicate if changes were made. The images or other third party material in this article are included in the article's Creative Commons licence, unless indicated otherwise in a credit line to the material. If material is not included in the article's Creative Commons licence and your intended use is not permitted by statutory regulation or exceeds the permitted use, you will need to obtain permission directly from the copyright holder. To view a copy of this licence, visit http://creativecommons.org/licenses/by/4.0/.

(C) The Author(s) 2020 